\title{
Morfologia endodôntica da raiz distal do primeiro molar inferior permanente
}

\author{
Milano, N.F.* \\ Manfro Jr., A.O.** \\ Marsiglia, M.** \\ Pan Nys, l.**
}

\section{RESUMO}

Os autores realizaram desgaste de raízes distais de primeiros molares inferiores permanentes, visando observar três aspectos: número de canais, secção dos mesmos e posiçāo do forame em relação ao vértice radicular.

\section{SUMMARY}

In this paper the autors studied the distal root of permanent $1^{\circ}$ lower molars acording 3 aspects: number of root canals, the anatomic profile of root canal and the position of apical foramen acording radicular apex.

\section{DESCRITORES}

\author{
ENDODONTIA ・ TRATAMENTO DE CANAIS・ANATOMIA
}

\section{INTRODUÇÃO}

No presente estudo foi proposta a pesquisa de:

a. número de canais

b. secção transversal do(s) canal(is)

c. posiçāo do forame em relaçāo ao vértice radicular.

Devido à discrepância de opiniōes entre os autores quanto ao número de canais nesta raiz, propusemo-nos a um estudo mais detalhado, visando a comprovação ou não dos trabalhos consultados.

Aproveitando as mesmas peças dentárias, realizamos o cateterismo do(s) canal(is) e pudemos com isso, obter a localização do forame principal em relação ao vertice radicular.

A seguir realizamos cortes transversais dividindo a raiz em terços, possibilitando a visualizaçāo do formato dos canais em cada terço da raiz.

\section{REVISÃO DA LITERATURA}

1. Quanto ao número de canais

Encontramos muita divergência entre os autores consultados quanto aos aspectos a serem observados no presente trabalho.

Autores como Diamond (17), Wheler (54) e Brescia (5), apenas mencionaram a presença de um canal.

$\mathrm{Na}$ grande maioria dos autores encontramos a preocupação em mencionar a presença de dois canais, todavia notamos que alguns apenas relataram ou admitiram a presença de dois canais, tais como Grossman (23) e (24), Holland (28), Sommer (50), Kraus (31), Kuttler (33), além de admitirem a presença de dois canais, tentam explicar o fato dizendo que isto poderia acontecer por existir uma raiz supra-numerária ou bifurcaçăo na mesma. Kuttler (33), Soler (49) e Valls (52) também preconizaram que a deposição de dentina secundária poderia transformar o canal distal em dois canais.

Os autores a seguir pesquisaram o tema que estamos estudando.

Hess (25), Giuntoli (18) e Lasala (34) concordam com a percentagem de $4 \%$ dos casos por eles estudados com dois canais.

Keller (30), Pucci (45), Kuttler (33) e Araújo (2), confirmando Green (22) en- contraram percentagens tais como $7,3 \%$, $13,5 \%$ e $14,25 \%$ respectivamente.

Okomura (38) estudando 468 peças encontrou $19,6 \%$ com dois canais. Skidmore (48) achou $28,9 \%$ e De Deus (13) $36 \%$ demonstrando percentual bem elevado. Ingle (29) em $8 \%$ dos casos com dois canais encontrou $5 \%$ com um forame e $3 \%$ com dois forames. Finalmente Cohen (9) afirmou que $33 \%$ dos casos por ele estudados apresentaram dois canais.

2. Quanto à localização do forame principal em relação ao vértice radicular

Baseados no princípio fundamental de que o respeito aos tecidos peri apicais é requisito para obtermos sucesso em endodontia, devemos estar atentos ao fato de que o forame principal não costuma estar no vértice da raiz.

\footnotetext{
* Professor Titular de Endodontia da F.O./UFRGS

** Ex-Alunos do Curso de Especialização em Endodontia da F.O./UFRGS
} 
Ocupam-se do assunto, Keller (30), Milano e cols. (37), concluindo que, respectivamente, em apenas $25,5 \%$ e $12,6 \%$ dos casos mostraram a abertura foraminal coincidindo com o vértice, predominando uma posiçāo excêntrica. Kuttler (32) dividiu o total de peças estudadas em dois grupos, por faixa etária. Na faixa de 18 a 25 anos encontrou $32 \%$ de forames cêntricos e na faixa com mais de 55 anos, apenas $20 \%$. Autores como Rothier (46), Cavada (7), Palmer (41), Pesce e Lima Machado (42), Cohen (9) e Paiva e Antoniazzi (39), concordam que o vértice anatómico apresentado na radiografia năo coincide com a realidade, dando-nos falsas interpretaçōes com referência ao limite apical dos canais. Todos esses autores apresentaram trabalhos relacionando peças dentárias com imagens radiográficas.

Quanto à distância da saída do forame ao vértice houve variaçōes nos achados, entre $0.5 \mathrm{~mm}$ até $03 \mathrm{~mm}$.

\section{Quanto à forma da secção}

transversal do(s) canal(is)

A importância deste item prende-se à importância da instrumentaçăo de todas as paredes do canal, cuja secção não costurna ser circular, como săo os instrumentos.

Não houve concordância entre os autores consultados quanto a este item.

Assim sendo, os canais foram classificados por Pucci (45), Sommer (50), Holland (28) apenas como amplo. Grossman (23), Hizatugu (27) qualificam de amplos e afunilados. Wheeler (53), Valls (52) e Leonardo (35) descrevem como amplos e achatados mesio-distalmente.

Outros como Hess (25), Wheler (54) e Soler (49) referiram como amplo e arredondado, sendo que os dois últimos autores admitiram a possibilidade de ser achatado.

Brescia (51), De Deus (13) e Cohen (9) relataram como achatado no sentido mésio-distal sendo que De Deus usa a denominação de ovalado em forma de rim. Araujo (1) denomina a secção como oval e Green (22) e Della Serra (16) como oitoide.

\section{PROPOSIÇÃO}

O propósito deste trabalho é o de estudar a freqüência da existência de dois canais na raiz distal dos primeiros molares inferiores permanentes, da localizaçāo e comportamento do eventual segundo canal, da secçāo transversal e finalmente, da distância do forame em relaçāo ao vértice radicular.

\section{MATERIAL E MÉTODO DE TRABALHO}

Foram utilizados 76 dentes extraídos, previamente imersos em solução de $\mathrm{NaOCl}$ a 2,5\%. Após a separaçāo da raiz distal fez-se o cateterismo do canal com limas tipo $\mathrm{K} \mathrm{n} \mathrm{n}^{\circ} 08$ e 10. Procurou-se discreto transpasse do forame principal para possibilitar sua localização em relação ao vértice radicular. Durante o cateterismo buscou-se localizar o eventual segundo canal.

Os 26 primeiros casos foram radiografados, mas em virtude do grande achatamento no sentido mésio-distal destas raízes, muitas as imagens obtidas deram falsa impressảo da existência de dois canais. Promoveu-se entảo o corte longitudinal de 8 peças, o que também nảo mostrou bons resultados. Nos 68 casos restantes, após o cateterismo efetuaramse secçōes transversais nas mesmas, o que permitiu, finalmente, constatarmos a existência ou não do segundo canal e o estudo morfológico da secção do(s) canal(is) em diferentes alturas da raiz.

\section{RESULTADOS}

\section{A. Quanto ao número de canais}

Em 50 casos encontrou-se um único canal em toda a extensāo da raiz. Ver tabela $n^{\circ} 1$.

\begin{tabular}{|c|c|c|c|c|}
\hline \multirow{2}{*}{$\begin{array}{c}\text { N: DE } \\
\text { RAIZES } \\
\text { EXAMINADAS }\end{array}$} & \multicolumn{4}{|c|}{ N: DE CANAIS } \\
\cline { 2 - 5 } & \multicolumn{2}{|c|}{$1 \mathrm{C}$} & \multicolumn{2}{c|}{ 2C } \\
\cline { 2 - 5 } & $\mathrm{N}:$ & $\%$ & $\mathrm{~N}:$ & $\%$ \\
\hline 76 & 50 & 65,8 & 26 & 34,2 \\
\hline
\end{tabular}

Em 26 casos observaram-se dois canais. Ver tabela $n^{\circ} 2$. Dentre os 26,14 casos apresentaram dois canais em toda a extensão; 4 casos mostraram no início um único canal para, no terço médio apresentar uma bifurcação, continuando com dois canais; 5 casos evidenciaram a bifurcaçāo à altura do terço apical; finalmente 3 casos mostraram dois de início, unindo-se no terço médio.

\section{B. Quanto a distância do forame ao vértice}

Houve uma grande variaçäo neste item. A distância variou de 0 a $3 \mathrm{~mm}$. Observou-se que, em 35 casos a distância foi de $0,5 \mathrm{~mm}$, em 19 casos foi de $1 \mathrm{~mm}$, em 3 casos foi de $1,5 \mathrm{~mm}$, em 7 casos foi de $2 \mathrm{~mm}$, em 1 caso foi de $3 \mathrm{~mm}$ e, finalmente, em 15 casos o forame encontrou-se no vértice. Ver tabela $n^{\circ} 5$.

\section{Posiçāo do forame quanto a localização da saída em relaçăo ao vértice radicular}

Em 15 casos a saída apresentou-se no vértice; em 6 casos a saída foi para mesial; em 6 casos a saída foi para mésiovestibular; em 4 casos a saída foi para vestibular; em 8 casos a saída foi para disto-vestibular; em 10 casos a saída foi para distal; em 14 casos a saída foi para disto-lingual; em 5 casos a saída foi para lingual; em 5 casos a saída foi para mesio-lingual. Ver tabela $n^{\circ} 6$.

\section{Quanto a secçăo transversal do(s) canal(is)}

D.1. Casos com um canal

Dos 50 casos onde foi encontrado somente um canal, 32 apresentaram a secção transversal com achatamento no sentido mésio-distal; 4 casos mostraram-se achatados no sentido mésio-distal no terço cervical, passando a ovoide a seguir; 5 casos iniciaram achatados no sentido mésio-distal no terço cervical, passando a circular nos terços subsequentes; 3 casos mostraram-se com secção ovoide em toda a extensão; 1 caso apresentou-se com secção ovoide no terço cervical, passando a circular nos terços seguintes; 1 caso mostrou-se de secção circular em toda a extensāo; 4 casos foram perdidos por ter sido corte longitudinal na raiz.

\section{D.2. Casos com dois canais}

Dos 26 casos encontrados com dois canais foram observadas 14 peças $(53,8 \%)$ com dois canais em toda a extensāo da raiz. Dentre estes, 3 mostraram-se circulares; 3 achatados mésio-distalmente passando a circular e finalmente, 2 casos, com um canal achatado mésiodistalmente e o outro circular. 4 peças fo-

TABELA Il: Raízes distas com dois canais (destrição da extensão longitudinal especifica de cada raíz estudada)

\begin{tabular}{|c|c|c|c|c|c|c|c|c|}
\hline & & \multicolumn{6}{|c|}{ RAIZ DISTAL COM 2 CANAIS } & TOTAL \\
\hline \multicolumn{2}{|c|}{$\begin{array}{l}\text { TODA A } \\
\text { EXTENSÃOO }\end{array}$} & \multicolumn{2}{|c|}{$\begin{array}{l}\text { SOMENTE NO } \\
1 / 3 \text { APICAL }\end{array}$} & \multicolumn{2}{|c|}{$\begin{array}{l}\text { A PARTIR DO } \\
\text { 1/3 MÉDIO }\end{array}$} & \multicolumn{2}{|c|}{$\begin{array}{l}\text { SOMENTE NO } \\
1 / 3 \text { CERVICAL }\end{array}$} & \\
\hline № & $\%$ & № & $\%$ & № & $\%$ & № & $\%$ & \\
\hline 14 & 53,8 & 05 & 19,2 & 04 & 15,3 & 03 & 11,5 & 26 \\
\hline
\end{tabular}


ram perdidas por ter sido feito o corte longitudinal.

Dos 5 casos onde houve bifurcação somente no terço apical, encontramos apenas 1 caso com ambos canais circulares; 2 casos mostraram distintamente, um canal ovoide e outro achatado no sentido mésio-distal; 1 caso mostrou ambos canais de secçäo ovoide.

Dos 4 casos onde a bifurcação ocorreu a partir do terço médio, encontrou-se 1 caso onde ambos mostraram achatamento mésio-distal; 2 casos com ambos canais com secção circular. A $4^{a}$ peça foi perdida por ter sido feito corte longitudinal. Finalmente em 3 casos foram achados 2 canais somente no terço cervical para a seguir, unirem-se em um so; destes 3 casos, 2 possuiam canais achatados mésio-distalmente e o outro foi perdido pelo corte longitudinal.

\section{DISCUSSÃO}

Procuramos, neste capítulo, comentar os achados da literatura, comparando-os com nossos resultados e tecendo os comentários cabíveis. Como nos capítulos anteriores, faremos a discussão em funçāo dos sub-capítulos.

1. Quanto ao número de canais

De acordo com a literatura, houve variaçōes quanto à freqüência de um segundo canal na raiz distal do $1^{\circ}$ molar inferior. A discrepância foi desde a inexistência de um segundo canal até a freqüência máxima encontrada de 35\% com dois canais. Muitas cifras intermediárias foram registradas como $4 \%, 7,3 \%$, $14,3 \%, 25 \%, 19,6 \%, 28,9 \%, 36 \%$ e alguns se referem à "mais dos casos".

Essa considerável variaçāo de cifras faz pensar em grande diferença nos métodos de trabalho levados a efeito.

Comparando-se os resultados encontrados na literatura com os do presente trabalho, que foi de $34,2 \%$, dá margem a afirmar-se que é significativa a presença

TABELA III: Secção transversal dos casos que apresentaram 1 canal

\begin{tabular}{|c|c|c|c|c|c|c|c|c|c|c|c|c|c|}
\hline $\begin{array}{c}\text { ACHAT ADO } \\
\text { M-D }\end{array}$ & $\begin{array}{c}\text { ACHATADO } \\
\text { M-D } \\
\begin{array}{c}\text { PAS SANDO } \\
\text { A OVÓIDE }\end{array}\end{array}$ & $\begin{array}{c}\text { ACHAT ADO } \\
\text { M-D } \\
\text { PASSANDO } \\
\text { A CIRCULAR }\end{array}$ & $\begin{array}{c}\text { OVÓIDE } \\
\text { PASS ANDO } \\
\text { CIRCULAR } \\
\text { A CIRCULAR }\end{array}$ & \multicolumn{2}{|c|}{ OVÓIDE } & \multicolumn{2}{|c|}{ CIRCULAR } & \multicolumn{2}{|c|}{ TOTAL } \\
\hline $\mathrm{N}:$ & $\%$ & $\mathrm{~N}:$ & $\%$ & $\mathrm{~N}:$ & $\%$ & $\mathrm{~N}:$ & $\%$ & $\mathrm{~N}:$ & $\%$ & $\mathrm{~N}:$ & $\%$ & $\mathrm{~N}:$ & $\%$ \\
\hline 32 & 69,5 & 4 & 8,6 & 5 & 10,8 & 1 & 2,1 & 3 & 6,5 & 1 & 2,1 & 4.6 & 100 \\
\hline
\end{tabular}

TABELA IV: Secção transversal dos casos que apresentarem dois canais

\begin{tabular}{|c|c|c|c|c|c|c|c|c|c|}
\hline \multicolumn{2}{|c|}{$\begin{array}{c}\text { ACH ATADO } \\
\text { M-D }\end{array}$} & $\begin{array}{c}\text { ACHAT ADO M-D } \\
\text { PASSANDO A } \\
\text { OVÓIDE }\end{array}$ & $\begin{array}{c}\text { ACHAT ADO M-D } \\
\text { PASSANDO A } \\
\text { CIRCULAR }\end{array}$ & \multicolumn{2}{c|}{ CIRCULAR } & \multicolumn{2}{c|}{ TOTAL } \\
\hline$\%$ & $\mathrm{~N}:$ & $\%$ & $\mathrm{~N}:$ & $\%$ & $\mathrm{~N}:$ & $\%$ & $\mathrm{~N}:$ & $\%$ & $\mathrm{~N}:$ \\
\hline & & & & & & & & & \\
36,3 & 16 & 4,5 & 2 & 34 & 15 & 25 & 11 & 100 & 44 \\
\hline
\end{tabular}

\section{OBS.:}

Os dados acima foram tabulados considerando cada canal distintamente, dentre os casos que apresentaram dois canais ( 22 casos $=44$ canais) e que a secção realizada foi a transversal, desprezando-se os casos de corte longitudinal.

de um segundo canal na raiz distal do $1^{\circ}$ molar inferior.

\section{Quanto à secção transversal}

a. Casos com um só canal

Chamou-nos a atenção pela propriedade, a denominação dada por alguns autores à forma da secção dos canais presentemente em estudo. Assim sendo, De Deus refere-se à forma de "rim", enquanto que Green e também Dela Serra classificam a secçāo como "oitóide".

No presente trabalho predominou o tipo achatado no sentido mésio-distal (60\%). Seguiu em freqüência o tipo achatado passando, após o terço cervical, à ovóide $(13,9 \%)$ e circular $(13,9 \%)$.

Entendemos como fundamental que seja conscientizada a secçāo achatada desses canais para quando da instrumentação, pois, sendo a secção do instrumento sempre circular e a secçăo do canal achatada, é forçoso admitir-se que durante a cinemática da instrumentação esse dado seja levado em conta, tornando- se fundamental o movimento de translaçāo para permitir a instrumentaçāo de todas as paredes do canal.

b. Casos com 2 canais

A literatura ocupa-se muito pouco da análise da secçăo transversal dos canais, quando sāo dois.

O presente trabalho deixa ver que năo há um predomínio de forma da secçāo transversal quando da existência de dois canais.

Eles podem ser totalmente achatados mésio-distalmente; podem também iniciar achatados e passarem a uma secçäo circular ou serem totalmente circulares ou ovóides.

\section{Quanto à posição do forame}

Neste item, cabe discutir a localização geográfica do forame, bem como quando nāo for no vértice, estabelecer a distância do forame ao vértice.

No que tange à localizaçăo, nossos achados se assemelharam à maioria dos autores que se ocuparam do assunto, ou

TABELA V: Distância do forame ao vértice da raiz.

\begin{tabular}{|c|c|c|c|c|c|c|c|c|c|c|c|c|c|c|c|c|}
\hline \multirow{2}{*}{$\begin{array}{l}\text { DISTÂNCIA } \\
\text { EM mm }\end{array}$} & \multicolumn{2}{|c|}{0} & \multicolumn{2}{|c|}{0,5} & \multicolumn{2}{|c|}{1,0} & \multicolumn{2}{|c|}{1,5} & \multicolumn{2}{|c|}{2,0} & \multicolumn{2}{|c|}{2,5} & \multicolumn{2}{|c|}{3,0} & \multicolumn{2}{|c|}{ TOTAL } \\
\hline & № & $\%$ & Ne & $\%$ & No & $\%$ & № & $\%$ & N: & $\%$ & № & $\%$ & № & $\%$ & No & $\%$ \\
\hline N. de casos & 15 & 18,75 & 35 & 43,75 & 19 & 23,75 & 3 & 3,75 & 7 & 8,75 & 0 & 0 & 1 & 1,25 & 80 & \\
\hline
\end{tabular}

OBS:

O total de casos não coincide com o número de rízes porque em algumas havia dois forames e em outras não se conseguiu atingir o forame, tendo-se desprezado para este item do trabalho.

TABELA VI: Localização do forame em relação ao vértice radicular.

\begin{tabular}{|c|c|c|c|c|c|c|c|c|c|}
\hline LOCALIZAÇÃO & ÁPICE & $\mathrm{M}$ & $\mathrm{MV}$ & $\mathrm{V}$ & $\mathrm{DV}$ & $\mathrm{D}$ & $\mathrm{DL}$ & $\mathrm{L}$ & $\mathrm{ML}$ \\
\hline N: DE CASOS & 15 & 6 & 6 & 4 & 8 & 10 & 14 & 5 & 5 \\
\hline PERCENTUAL & 24,6 & 8,2 & 8,2 & 5,4 & 10,9 & 9,5 & 19,1 & 6,8 & 6,8 \\
\hline
\end{tabular}

seja, em apenas $24,6 \%$ dos casos o forame coincidiu com o vértice da raiz. Em $39,9 \%$ a tendência foi para distal (D - DL DV) e em $23,2 \%$ foi para mesial (M - ML MV). 
A distância do forame ao vértice foi variável, desde $0 \mathrm{~mm}$ até $2,5 \mathrm{~mm}$. A predominância foi de $0,5 \mathrm{~mm}(43,75 \%)$ e $1 \mathrm{~mm}$ $(23,75 \%)$.

Esse achado é fundamental quando da condutometria, fato ressaltado por vários autores.

\section{CONCLUSÕES}

1. É considerável a freqüência de dois canais na raiz distal do $1^{\circ}$ molar inferior $(34,2 \%)$. Por conta disso fica determinada a importância de o endodontista pesquisar atentamente a possibilidade de existência do dito canal.

2. Quanto à secçāo transversal é significante a freqüência da forma achatada mésio-distalmente (60\%), especialmente quando existe um só canal. Quando há dois canais, a secçäo é variável, podendo ser achatada, ovóide ou circular.

3. É predominante a posiçāo "nāo cêntrica" do forame.

Em apenas $24,6 \%$ dos casos o forame coincide com o vértice da raiz.

A distância do forame ao vértice nos casos ectópicos é predominantemente de $0,5 \mathrm{~mm}$ a $1 \mathrm{~mm}$.

\section{REFERÊNCIAS BIBLIOGRÁFICAS}

1. ARAUJO, M.J. Anatomia e biomecânica dos canais radiculares. Rev. Bras. Odontol., v. 14, p. 231-255, 1956.

2. ARAUJO, M.J.; ARAUJO M.C. Anatomia da cavidade pulpar. Rev. Bras. Odontol., v. 26, n०147, p. 338-368, mai/jun 1967.

3. BERBERT, A.; BRAMANTE; CLÓVIS MONTEIRO; BERNARDINELI; NORBERTI. Endodontia prática. Sāo Paulo: Savier, 1980. $110 \mathrm{p}$.

4. BRAMANTE, C.; BERBERT, A. A critical evaluation of some of methods of the termining teeth lenght. Oral Surg. Oral Med. Oral Patho!., v. 37, p. 463-476, 1974.

5. BRESCIA, N.J. Applied dental anatomy. St. Louis: Mosby, 1961 p. 59.

6. BURCH, J.C.; HULLEN, S. The relationship of the apical foramen to the anatomic apex of the tooth. Oral Surg. Oral Med. Oral Pathol.; v. 34, p. 262-268, 1972.

7. CAVADA, L.C. Verificaçāo radiográfica da relaçāo forame e ápice radicular. Pelotas, 1977. Tese.

8. CAUDURO, H. Manual prático de endodontia. 2. ed. Porto Alegre: Ed. R.G.O., 1969. p. 8.

9. COHEN, S.; BURNS, R.C. Caminhos da polpa. 2. ed. Rio de Janeiro: Guanabara Koogan, 1982. p. 96-97.

10. COOLIDGE, E.D. Anatomy of the root apex in relation to the treatment problems. J. Am. Dent. Assoc., v. 16, n 8, p. 456-465, 1929.

11. COOLIDGE, E.D.; KESEL, R.G. Endodontia. Philadelphia: Lea \& Febiger, 1950. p. 132.

12. COOLIDGE, E.D.; KESEL, R.G. Manual de endodontia. Buenos Aires: Bibliográfica Argentina, 1957. cap. 7: Anatomia de los conductos radiculares en relacion a la remocion pulpar y a la obturacion. p. 133.
13. DE DEUS, Q.D. Endodontia. Belo Horizonte: Odontomédica \& Jurídica, 1973. cap. 3: Topografia da cavidade pulpar e do periápice. p. 71-72.

14. DE DEUS, Q.D. 3. ed. Rio de Janeiro: Medsi, 1982. cap. 5: Alteraçōes da polpa dental. p. 119-152.

15. DELLA SERRA, O.; FERREIRA, F.V. Anatomia dental 2. ed. Sāo Paulo: Artes Médicas, 1975. cap. 9: Cavidade pulpar. p. 217-218.

16. DELLA SERRA, O. 3. ed. Sāo Paulo: Artes Médicas, 1981. cap. 9: Cavidade pulpar. p. 217-218.

17. DIAMOND, M. Anatomia dental. Buenos Aires: Uteha, 1940. cap. 16: Camara y canalis pulpares. p. 199.

18. GIUNTOLI, A. Contribuicion al estudio de los conductos radiculares. Local: A.F.O., 1955.

19. GREEN, D. Morphology of the pulp cavity of the permanent teeth. Oral Surg. Oral Med. Oral Pathol. v. 8, $n^{\circ}$ 7. p. $749-759$, july, 1955.

20. GREEN, D. Stereomicroscopic study of the root apices of $\mathbf{4 0 0}$ maxilary and mandibular anterior teeth. Oral Surg. Oral Med. Oral Pathol. v. 9, n० 11. p. 1224-1232, nov., 1956.

21. GREEN, D. Stereomicroscopic study of the apices of maxilary and mandibular posterior teeth. Oral Surg. Oral Med. Oral Pathol. v. $13, n^{\circ}$ 6. p. $728-733$, jun., 1960.

22. GREEN, E.N. Microscopic investigation of root canal diameters. J. Am. Dent. Assoc. v. 57. p. 636-655, 1958.

23. GROSSMAN, L.I. Endodontic practice. 5. ed. Philadelphia: Lea \& Febiger, 1952. p. 402

24. GROSSMAN, L.I. Endodontia prática. 8. ed Rio de Janeiro: Guanabara Koogan, 1976.

25. HESS, W. Formation of root canals in human teeth. J. Am. Dent. Assoc. v. 8. p. 790-832, 1921.

26. HIBBARD, Journal of dentistry for children. v. 24. p. 250-257, 1957.

27. HIZATUGO, R.G.; VALDRIGUI, L. Endodontia. Sāo Paulo, Aloisi, p. 70-92, 1974.

28. HOLLAND, R. et al. Endodontia: Manual para alunos de graduaçāo. Araçatuba: Faculdade de Odontologia, 1974. cap. 1: Aberturas coronárias. p. 11.

29. INGLE, J.I.; BEVERIDGE, E.E. Endodontia 2. ed. Rio de Janeiro: Interamericana, 1979, $731 \mathrm{p.}$.

30. KELLER, O. The anatomy canals of the human dentition according to the classification methods. Dental Cosmos. v. 71. p. 330, 1929.

31. KRAUS, BERTRAM S.; JORDAN, RONALD E.; ABRAMS, LEONARD. Dental anatomy and occlusion. Baltimore, Williams and wilkins, 1969. cap. 1: Anatomy. p. 94-110.

32. KUTTLER, Y. Microscopic investigation of root apices. J. Am. Dent. Assoc. v. 50, $n^{\circ}$ 19. p. 344-352, 1955.

33. KUTTLER, Y. Endodoncia práctica. México: Alpha, 1961. 303 p.

34. LASALA, A. Endodoncia. 2. ed. Carácas: Cromotip, 1971. p. 12.

35. LEONARDO, M.R. Aspectos anatômicos da cavidade pulpar. In: LEONARDO, M.; LEAL, J.N.; SIMŌES FILHOS, ARIANO PENTEADO. Endodontia: Tratamento dos canais radiculares. Sāo Paulo: Panamericana, 1982. p. 138

36. MAISTO, O. Endodoncia. Buenos Aires:
Mundi, 1969. $344 \mathrm{p}$.

37. MILANO, N.F. et al. A localizaçāo do forame principal. Rev. Gaúcha Odontol., v. 31, n०3. p. 220-4, 1983.

38. OKOMURA, T. Anatomy of the root canals. J. Am. Dent. Assoc. v. 14. p. 632-636, Apr. 1967.

39. PAIVA, J.G.; ANTONIAZZI, J.H. Endodontia. Rio de Janeiro: Artes Médicas, 1984. 867 p.

40. VARELLA, J.F.; PAIVA, J.G. Manual de Endodontia. 2. ed. Sāo Paulo: Atheneu, 1969. $222 p$.

41. PALMER, M.J. Position of the apical foramen in relation endodontic therapy. J. Canad. Dent. Assoc. v. 37, $n^{\circ}$ 8. p. 305-308, 1971.

42. PESCE, H.F.; LIAM MACHADO, M.E. Estudo da regiāo apical de dentes tratados endodonticamente até o vértice radiográfico na raiz. Rev. Assoc. Paul. Cir. Dent. v. 35, $n^{\circ} 6$, p. 534-537, nov/dez., 1981.

43. PICOSSE, M. Anatomia dentária. 2. ed. Sāo Paulo, Savier, 1977. cap. 5: Morfologia dos dentes permanentes. p. 85-166.

44. PRECIADO, V. Manual de Endodontia Clínica. Guadalajara: Cuellar Ediciones, 1975, p. 92.

45. PUCCl, F.M. Conductos Radiculares. Montevideo: Médico Quirúrgica, 1945. v. 2, p. 332-7.

46. ROTHIER DUARTE, A. Estudo radiográfico in vitro de canais radiculares obturados até o limite foraminal. Rio de Janeiro, 1975.

47. SHOJI, Y. Endodoncia Sistemática. Berlim, quintessenz, 1974. cap. 10, p. 10-4: Anatomia práctica de los clientes para el tratamiento radicular. p. 73.

48. SKIDMORE, A.F.; BJORDAL, A.M. Root canal morphology of the human mandibular first molar. Oral Surg. Oral Med. Oral Pathol. v. 33, n०5. p. 778-784, jul/dec, 1971.

49. SOLER, R.M.; SHOCRON, N.L. Endodoncia. Cordoba: La Médica, 1957. 378 p..

50. SOMMER, R.F.; OSTRANDER, F.D.; CROWLEY, M.C. Clinical endodontics. 2. ed. Philadelphia: Saunders, 1961. cap. 2: Endodoncia. p. 6-55.

51. STILLSONS, W.C. Dental anatomy. Philadelphia: Saunders, 1929.

52. VALLS, L.A. Endodontia. Havana: Inst. Cubano do Livro, 1977. p. 62.

53. WHEELER, R.C. A text book of dental anatomy and phisiology. 4. ed. Philadelphia: Saunders, 1965.

54. WHEELER, R.C. Dental anatomy phisiology and oclusion. 2. ed. Philadelphia: Saunders, 1953. 